\title{
Gaps in electronic Trial Master File (eTMF) implementation: A study in the organization case
}

\author{
Oraya Wisawapaisarn ${ }^{1 *}$, Pitipong Yodmongkol ${ }^{2}$ \\ ${ }^{1,2}$ College of Arts, Media and Technology, Chiang Mai University, Chiang Mai, Thailand
}

\author{
Index Terms \\ Trial Master File \\ Enterprise Content Management \\ System Implementation
}

Received: 15 August 2017

Accepted: 20 September 2017

Published: 16 October 2017

\begin{abstract}
The concept of this study was based on concerns about the implementation of the eTMF system. This case study aimed to identify and gain an in-depth understanding of the gaps in eTMF system implementation for organizations in Thailand. Interviews followed a semi-structured questionnaire with fourteen users of the eTMF system, which were conducted to collect users' opinions concerning eTMF system satisfaction, tool (named master list) application, eTMF management processes complexity, and user knowledge. Besides, the survey was conducted to assess user knowledge on the master list application and eTMF management processes. The interview findings revealed that the unsatisfied search function of the eTMF system was the highest-ranking gap. The second and third-ranking gaps were the lack of understanding and the complexity of the master list, respectively. Lack of knowledge concerning the master list and eTMF system functions was ranked as the fourth-ranking gap. Lastly, repeating the processes of eTMF was the fifth-ranking gap. Also, the results of the user knowledge assessment confirmed the lack of knowledge in eTMF management processes and the master list. Determining the critical gaps in system implementation would be useful for the application of suitable solutions. Future studies will utilize practices, tools, and guidelines developed for success in system implementation with a knowledge management approach. An efficient knowledge management program allows the utilization of knowledge, including improving users' productivity and satisfaction.
\end{abstract}

(C) 2017 The Author(s). Published by TAF Publishing.

\section{INTRODUCTION}

Many organizations are in the process of deciding and implementing ECM to administer content in their organizations. The term ECM refers to systematic information collection and the organization used by a designated audience, both inside and outside the organization. Besides, it includes the technology, tools, and methods that involve multiple activities such as capturing, managing, storing, preserving, and delivering content within and across organizations [1, 2]. Many researchers describe ECM as the evolution of document management, record management, workflow management, and web Content Management Systems (CMS) [3]. ECM supports and enhances many other business functions within organizations [4]. It aims to reduce the costs of creating, managing, and distributing content, as well as ensures that the content supports organizational needs. Therefore, businesses should adopt and implement ECM that aligns with their corporate objectives. ECM includes multiple dimensions such as technology and the interaction of business with content, people, processes, and tools $[3,5]$. Furthermore, the ECM system life cycle and strategic managerial aspects are elements of ECM. The ECM system life cycle consists of adoption, acquisition, evolution, and evaluation, while strategic managerial aspects include change management and management commitment [3]. Challenges in ECM implementation may cause difficulties in business processes and the loss of valuable informa-

\footnotetext{
${ }^{*}$ Corresponding author: Oraya Wisawapaisarn

†Email: orayawisa@gmail.com
} 
tion and knowledge. Determining the dimensions of EMC is the key to understanding challenges and managing them for advantages in business [5]. Besides, personal aspects such as user experience and motivation may influence the procedure of ECM and its effects [6]. Although many challenges can affect ECM implementation, organizations should apply techniques and technologies to encourage the implementation and application of successful ECM [7].

The concept of this study was based on concerns about the implementation of the existing ECM system, called electronic eTMF system, in the organization case in Thailand. The eTMF system is ECM for managing clinical trial documents. eTMF system users have to file documents using clinical trials in the eTMF system correctly as per regulatory requirements. Document-filing completeness and accuracy on required processing time is the performance of the organization case. However, this organization case does not meet this performance. This study was a case study that focused on eTMF implementation through ECM dimensions: technology (system), processes, tool, and people aspects. This study aimed to identify and understand the gaps in eTMF system implementation in the organization case. The research questions that guided the study are as follows: 1) What are the gaps in eTMF system implementation in the organization case?

2) What is the most critical gap in eTMF system implementation in the organization case?

\section{LITERATURE REVIEW}

TMF plays a significant role in clinical trials to comply with applicable international and local regulatory requirements. TMF is a compilation of essential documents and other content that authorizes the evaluation of conducting clinical trials and the quality of data. Such documents illustrate whether or not investigators, sponsors, and monitors are in compliance with the standards of Good Clinical Practice (GCP) and regulatory requirements. The International Conference on Harmonization Good Clinical Practice (ICHGCP) Guideline provides master files consisting of a list of essential documents obtained during the conduction of the trial required for TMF filing and their purpose in the trial [8].

Organizations, where clinical trials are conducted, actively use TMF to collect and maintain various essential documents and contents obtained before the start of clinical trials, during the conduction of clinical trials, and in the post-trial period. TMF should be ready and available for regulatory agency inspection [9]. Traditionally, TMF involves paper-based documents filed in physical file cabinets. The complexity of managing clinical trial documents, tasks, and processes using paper-based content can introduce errors and create a high degree of variability and inconsistency. Organizations face challenges in managing TMF, such as difficulty in compliance with regulatory requirements, the high cost of TMF management, the time required for document management, inefficient processes to contribute, and insufficient resources. Therefore, many organizations involving clinical trials implement use of electronic eTMF. eTMF covers automated processes and workflow to collect, classify, index, archive, and report on documents and content. Examples of eTMF implementation are the enhancement of document quality, growth in regulation, and reduction of auditing and reporting costs. It is crucial to empower organizations for business productivity, cost savings, and shorten product development timelines [10].

Implementing eTMF introduces challenges regarding corresponding processes, security issues, TMF accessibility and authenticity, user-unfriendliness, and file formatting, including TMF completeness and accuracy. The European Medicine Agency's (EMA) reflection paper on eTMF reported on eTMF users' inability to locate requested documents. Such problems indicate inappropriate eTMF structure, insufficient training, and the lack of system usability. The reflection paper also suggested that the functionality of eTMF should offer functions to upload files, role-based permissions, audit trailing, and lock documents, covering additional store metadata. Besides, using such systems also requires similar procedures for validation, training, management, and change of control [11]. Furthermore, the use of eTMF presents additional problems during inspection and audit. For example, the inspector and auditor reported the failure of effective Quality Control on documents in the eTMF system, which found discrepancies such as missing pages, incorrect documents, and poor-quality scans. Sometimes, there was false labeling of files in the eTMF system [10]. The results of developing a risk-based approach for quality assessments of eTMF demonstrated missing documents in the eTMF system and deficiencies in quality control checks [12].

\section{METHODOLOGY}

This study identified gaps in eTMF system implementation. A review of available literature identified the 
gaps and challenges. Investigation of existing ECM systems in multiple industries was performed. Interviews with users of the eTMF system were also conducted to collect users' opinions on eTMF system satisfaction, tool (named master list) application, eTMF management processes complexity, and user knowledge. Besides, a questionnaire survey was conducted to assess user knowledge on master list application and eTMF management processes.

\section{A. Interview - Gap Identification in eTMF System Imple- mentation}

A semi-structured questionnaire was developed based on the literature review. The organization case report was used in interviews. The questionnaire consists of four major parts:

1) eTMF system satisfaction.

2) Master list application.

3) eTMF management processes complexity.

4) User knowledge.

A test of the survey was conducted on five users to improve the clarity and order of questions. All five users involved in the pre-test understood the questions and had no negative comments concerning the questionnaire. The final questionnaire is detailed in Appendix A.

In this stage of the research, interviews with fourteen interviewees from the organization case in Thailand were conducted in June 2017. The interviewees were eTMF system users with at least one month's experience in the eTMF system, master list application, and eTMF management processes. All fourteen interviews were arranged with verbal consent obtained from all users. The topics of discussion were delivered to users before conducting interviews. Details and descriptions of users' opinions on the four parts were collected. Notes were taken during conversations. Users were requested to rate system satisfaction, master list application, eTMF management processes complexity, and user knowledge on a 5-point scale (1 = extremely bad, 2 = slightly bad, 3 = neither, 4 = slightly good, and $5=$ extremely good). Also, they were asked to explain gaps and provide suggestions for bridging the existing gaps in each part. The length of interviews was approximately 30 minutes. Triangulation techniques were used to confirm internal validity by combining the observational fieldwork and interview data. Respondent validation confirmed the interpretation of findings by cross-checking the interim results of the study with the interviews [13]. All fourteen users were cross-checked during the period of data collection to provide feedback on accuracy and interpretation. Also, a final talk was performed after the interpretation of all the data findings so that users could obtain feedback on interpreted results. The details of responses were filtered to exclude any irrelevant results.

\section{B. Questionnaire Survey - User Knowledge Assessment}

The questionnaire survey aimed to assess user knowledge on master list application and eTMF management processes. The evaluation consists of two sections; the first section investigated knowledge on eTMF management processes, and the second part evaluated knowledge on master list application. A preliminary assessment was conducted on five users to improve the clarity and order of questions. All five users involved in the pre-test understood the questions and had no comments to make concerning the questionnaire. The final questionnaire survey is detailed in Appendix B. A total of fourteen users finished the interviews and completed the assessment via the online survey between June and July 2017. The results from the evaluation are important to point out users' knowledge of master list and eTMF management processes, including training needs.

\section{RESULTS}

\section{A. Interview Findings: System User Demographic Infor- mation}

Responses from a total of fourteen users were collected. Table 1 shows the system user demographic information. Seven users used the system less than three days per week, while the other seven users used the system 3-5 days per week. There were three types of system users consisting of document indexer, document approver, and both document indexer and approver. The majority of users were both document indexer and approver (Six users), followed by document indexer (Four users), and document approver (Four users). Most users (Nine users) typically used the master list as guidance for the indexing and approving of documents. Two users always used the master list to ensure that they correctly indexed and approved documents. Three users never used the master list; two users were new users, while a third user did not understand how to use the master list. 
TABLE 1

SYSTEM USER DEMOGRAPHIC INFORMATION

\begin{tabular}{llc}
\hline \hline System User Demographic Information & & Total \\
\hline System use frequency & $<3$ days per week & 7 \\
& $3-5$ days per week & 7 \\
& $>5$ days per week & 0 \\
System user type & Document Indexer & 4 \\
& Document Approver & 4 \\
\multirow{3}{*}{ Master list use frequency } & Both document indexer and approver & 6 \\
& Always & 2 \\
& Sometimes & 9 \\
\hline \hline
\end{tabular}

\section{B. Interview Findings: Gap Identification in eTMF System Implementation}

Interview findings were analyzed using two methods. The first method was quantitative analysis for rating the score of gaps in four major parts in eTMF system implementation. The second method was qualitative content analysis that interpreted details and descriptions of gap identification for each part.

Figure 1 shows the rating score with a 5-point scale of four sections in eTMF implementation:

1) eTMF system satisfaction

2) Master list application

3) eTMF management processes complexity.

4) User knowledge.

The average rating score for each part was calculated from all users. The score ranged from 1 to 5 ( $1=$ extremely bad, 2 = slightly bad, $3=$ neither, $4=$ slightly good, and $5=$ extremely good). The lower bars represent the rating score of users' opinions, while the top bars refer to the score of the gap.

Each gap shows a different score for the highest score (5 points) and rating score of users' opinions. A high score of gap represents the top ranking.

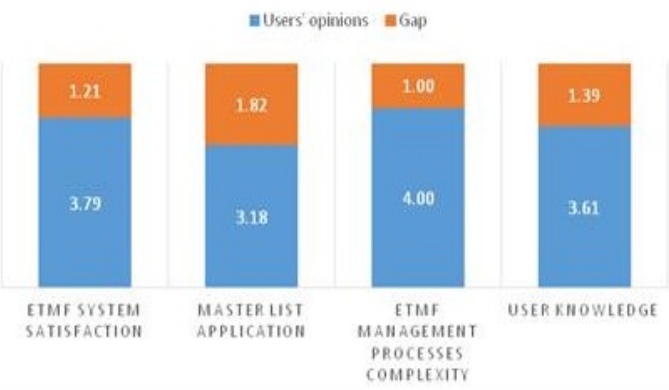

Fig. 1. Score of gap in eTMF system implementation ISSN: 2414-3103
The results from Figure 1 show that a rating of a gap in master list application (1.82) was the highest score. The score of the gap in user knowledge (1.39) was ranked as the second highest score, followed by the gap in eTMF system satisfaction (1.21) and eTMF management processes complexity (1.00). Details and descriptions of each gap in eTMF system implementation are described below.

\section{Master list application (Score of gap: 1.82)}

The master list is the material that guides a user in classifying the documents used in a clinical trial. This material is an Excel file that contains lists and specifications of all essential and non-essential documents. For example, details include the role and responsibility of the user, type, subtype, classification of each document, and documents required wet ink, etc. The primary reason for failure to utilize the master list was the lack of clear understanding of the details of the master list that linked to compliance with document filing. Users did not understand the purpose of the document reported by eight users. The lack of clear understanding also led to different users having different interpretations of the purpose of the document. A different interpretation might cause a discrepancy in document filing.

Other reasons for failure to utilize the master list were complexity details and feature of the Excel file reported by seven and five users, respectively. Users pointed out that the current elements of the master list were challenging and required simplification. The complicated master list caused users to not naturally understand the importance of this information in the master list. Also, they did not know how to apply the master list. Therefore, users needed time for master list application. Because of these gaps, users recommended that the master list should also

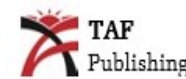


provide the purpose of the document together with an example. Also, a few users advised simplification of the master list to be more user-friendly. Lastly, training was needed on how to utilize the master list efficiently.

\section{User knowledge (Score of gap: 1.39)}

Lack of knowledge on the master list and eTMF system functions among users attributed to a lack of specific training on the master list and eTMF system. Six users reported that they had a lack of knowledge on the master list application. For example, six users stated that they could not classify some documents. Among these users, two indicated that they could not utilize information in the master list efficiently. Other gaps dealt with the knowledge and skills of users to use system functions effectively, such as the search function.

Five and three users recommended refresher training and focus group discussion, respectively, for improving their knowledge and understanding. Furthermore, users needed brief instructions to support system function utilization. On the other hand, many users (Eight users) had a positive attitude towards the system, including associated tools and eTMF management processes. The users' positive attitudes indicated an opportunity for improvement.

\section{System satisfaction (Score of gap: 1.21)}

A majority of users (ten users) reported that the search function of the eTMF system was not satisfactory. These users took a long time to search documents, although they used search keywords. When they used the search function, the system showed results with plenty of documents related to the keywords searched. However, a feature of the system was not exploited; it presented results of documents with the same title. Therefore, users could not identify what documents were needed. Consequently, they had to access each document to view content for proper identification.

Four users reported the eTMF System sometimes was slow, which caused frustration among users. Besides, the system account usually worked very well and reliably, but it sometimes had poor performance, as reported by three users. These users mentioned that the server and internet speed might be partly to blame for the slow performance. However, the main operative system was working well. Three users stated that the document uploading function did not work all the time or appropriately. Sometimes, documents did not come through. Based on gaps in system satisfaction, additional training is needed to exploit the eTMF system fully.

\section{eTMF management processes complexity (Score of gap: 1.00)}

All fourteen users had a positive attitude towards eTMF management processes. Although attitudes were positive, five users needed better methods. Among these five users, there was agreement that the processes were not complicated, although they reported that some processes were repeated and could be simplified. For example, an owner of the document classified the document and sent such document to a document indexer for uploading into the system. After that, the system sent an assignment back to the owner for approval. The document owner could not fully complete assigned tasks in one process. Furthermore, a few processes required lots of manual work and preparation, as reported by three users. Such processes involved the material named transmittal form completed by the document owner, which could make an error of discrepancy information and delay process transfer. Table 2 shows users' ranking of the five most prevalent gaps in eTMF system implementation. The results present unsatisfied search function of the eTMF system, which was the highest ranking gap.

TABLE 2

USERS' RANKING OF GAPS IN ETMF SYSTEM IMPLEMENTATION

\begin{tabular}{llc}
\hline \hline Part of eTMF Implementation & Gap Description & Rank \\
\hline eTMF system satisfaction & Unsatisfied search function of the eTMF system & 1 \\
Master list application & Lack of clear understanding of details in the master list & 2 \\
Master list application & Complexity of details and features of the master list & 3 \\
User knowledge & Lack of knowledge on the master list and eTMF system functions & 4 \\
eTMF management processes complexity & Repeated eTMF management processes & 5 \\
\hline \hline
\end{tabular}


The second and third ranking gaps were master list application; lack of clear understanding of details in the master list and complexity details, and feature of the master list. Lack of knowledge on the master list and eTMF system functions was ranked as the fourth ranking gap. Lastly, repeated processes of eTMF were the fifth ranking gap.

\section{User knowledge assessment}

A total of fourteen users completed user knowledge assessment on eTMF management processes and the master list. Only one user (7.14\%) could define eTMF management processes, while six users $(42.86 \%)$ knew the processing time for eTMF management processes (Table 3).

TABLE 3

USER KNOWLEDGE ASSESSMENT ON ETMF MANAGEMENT PROCESSES

\begin{tabular}{llcc}
\hline \hline Part of eTMF Implementation & Knowledge Assessment & Total & $\%$ \\
\hline eTMF management processes & Defining eTMF management processes & 1 & 7.14 \\
& Defining processing time & 6 & 42.86 \\
\hline \hline
\end{tabular}

TABLE 4

USER KNOWLEDGE ASSESSMENT ON ETMF MANAGEMENT PROCESSES

\begin{tabular}{llcc}
\hline \hline Part of eTMF Implementation & Knowledge Assessment & Total & $\%$ \\
\hline Master List & Master list function & & \\
& 1-3 Functions & 12 & 85.71 \\
& 4-6 Functions & 2 & 14.29 \\
& 7-9 Functions & 0 & 0.00 \\
& Defining "Attribute" of document in the master list & 10 & 71.43 \\
& Defining "Wet ink/ original file requirement" in the master list & 10 & 71.43 \\
& Defining definition/ purpose of document & & \\
& 1-8 Document/s & 6 & 42.86 \\
& 9-16 Documents & 5 & 35.71 \\
& 17-24 Documents & 3 & 21.43 \\
\hline \hline
\end{tabular}

The results from Table 4 show user knowledge assessment on the master list. The master list consists of nine functions. No users (14 users, 100\%) knew how to use all nine master list functions. Almost all users (12 users, 85.71\%) knew how to use 1-3 functions. Only a few users ( 2 users, 14.29\%) knew how to use 4-6 functions out of the nine master list functions. There was no user who knew how to use 7-9 master list functions.

Ten users (71.43\%) failed to define the attributes of each document and wet ink/ original file requirement in the master list.

No users (14 users, 100\%) could define the definition/purpose of all twenty-four documents. Six users (42.86\%) could define the definition/purpose of 1-8 documents. Five users $(35.71 \%)$ could define the definition/purpose of 9-16 documents. Three users (21.43\%) could define the definition/purpose of 17-24 documents out of 24 documents.

\section{DISCUSSION}

Concerning ECM literature, [3] proposed a conceptual ECM framework consisting of four ECM dimensions, ECM system lifecycle, and strategic managerial aspects based on ECM literature [3]. This study focused on ECM dimensions as technology (system), processes, tool, and people aspects. Grund and Mielebacher [11] presented an example of the eTMF management processes involving core eTMF system requirements [11]. The interview findings of this study illustrated gaps in eTMF system implementation. Also, the results of user knowledge assessment confirmed the lack of knowledge in eTMF management processes and the master list. Knowledge involves all of the processes of eTMF management, associated tools, and eTMF system. If users have a lack of clear understanding of the purpose of each essential document used in the clinical trial, eTMF 
management process flow, associated tool application, and functional system, the lack of knowledge leads organizational performance. Therefore, concentrating on critical knowledge is crucial for identifying the knowledge areas that need the most attention or improvement. Figure 2 shows identified critical knowledge of eTMF system management processes, associated tools, and eTMF system based on the organization case findings.

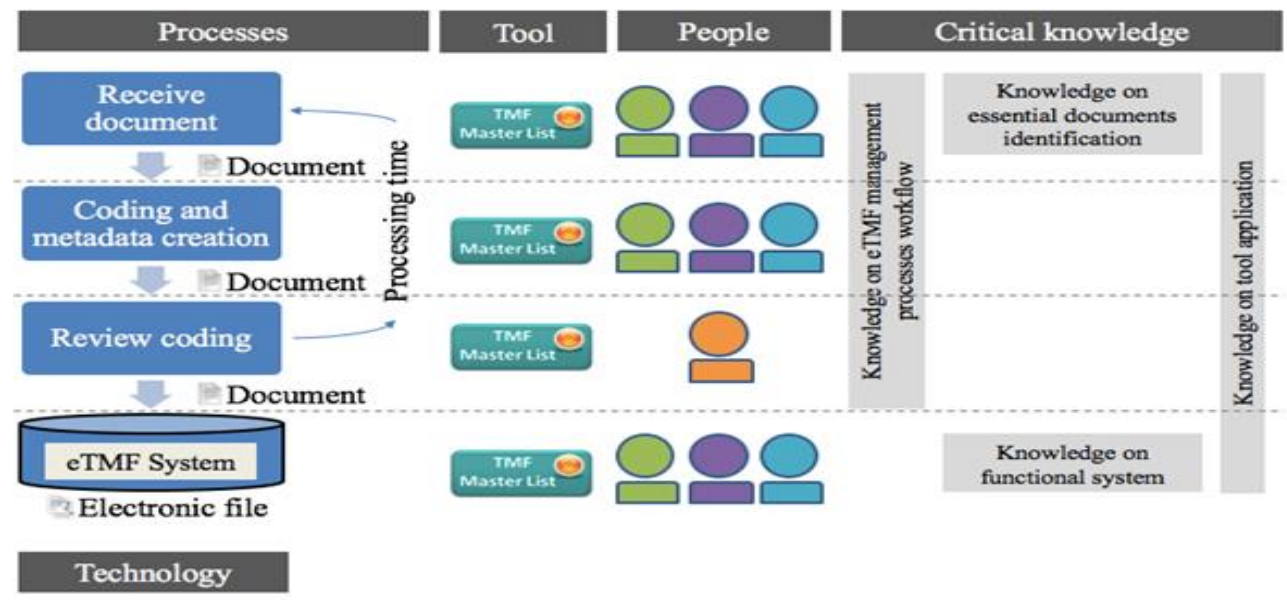

Fig. 2 . Identified critical knowledge

Firstly, the interview findings and knowledge assessment of essential documents demonstrated that users did not understand all of the purposes and definitions of essential documents. This challenge may be one of the causes of missing eTMF, including a lack of eTMF completeness and accuracy. Knowledge of essential document identification is essential for all processes of eTMF management. Essential document identification refers to users understanding the purpose or definition of each essential document used in the clinical trial. Then, they classify such documents with the correct code. This knowledge improves compliance in document filing as per regulatory requirements, including enhancement of eTMF completeness and accuracy.

Secondly, interview findings and knowledge assessment of essential eTMF management processes illustrated that users were not entirely knowledgeable in all processes, and were not aware of the processing time. This lack of understanding of processes may be a result of unawareness for users' responsibility in each process. Besides, not being aware of the processing time may lead to non-compliance of document filing; users file documents in the eTMF overdue timeline. Users require knowledge of eTMF management processes and workflow. Knowledge on processes workflow refers to users' certainty and awareness of all processes, including the responsibilities for each process and processing time. This knowledge encourages users to know what tasks they have to complete for each process.
Also, it improves compliance for document filing in a timely manner. [14, 15]

Thirdly, interview findings and knowledge assessment on the associated tool named the master list showed that some users had never applied this tool for document classification and filing. Moreover, most users used this tool but did not understand how to use the tool efficiently. The master list is a guidance tool for identifying essential documents used in the clinical trials and providing purposes or definitions including required information of documents. Besides, the master list provides instructions for entering necessary information in the documents in the eTMF system. Knowledge on tool application is one of the critical factors. This knowledge defines users' understanding of the purpose of the tool, including how to efficiently and correctly apply guidance in the tool for document filing in the eTMF system. The efficiency of the tool application is a result of eTMF completeness and accuracy with data integrity.

Lastly, the interview findings demonstrated that users did not know how to use various functions of the eTMF system. Consequently, they took time to search for documents in the system. Even if the feature of the system is one of the factors of user-unfriendliness, knowledge on functional system supports users to work through the system easier. This knowledge defines users' knowledge of the purpose of system functions, and understand how to apply 
such functions. The advantage of this knowledge is the capability of users to work with a vast array of documents via the system efficiently.

Research on the challenges and solutions of eTMF system implementation is needed. Further, determining the critical factors for system implementation would be most useful. User knowledge was the key element to deal with system implementation for this study. Therefore, knowledge management programs for the development of knowledge are crucial for resolving this challenge. However, user knowledge alone is not sufficient for the success of system implementation. Appropriate strategy, associated tools, and standard practices, including pre- and postimplementation training programs, are crucial for improving implementation.

\section{CONCLUSION AND RECOMMENDATIONS}

This paper described gaps in the ECM system called eTMF system of the organization case. The results based on interviews and user knowledge assessment highlighted the substantial gap in people with personal knowledge. System, processes, and tools are other gaps that involve system implementation. Firstly, the organization case highlighted the lack of knowledge in system, processes, and tools. Therefore, users require training programs after application and focus group discussion for better experience in the functional system, processes, and associated tools. Group discussion minimizes issues of different people having different interpretations and standardized practices. The organization has to consider the best training strategies and programs for system users' efficiency.

Secondly, the complexity of the system, processes, and tools is a gap in system implementation. Motivating users to deal with an unfriendly system, complex processes, and complicated tools is challenging and may lead to change resistance. The organization has to develop potential tools, practices, and guidelines to support system implementation.

Determining the critical gaps in system implementation is useful so that the organization understands the challenges and needs of users. Proper solutions are required based on such problems and requirements. This study supported the organization case to identify gaps and consider suitable solutions for system implementation. Future study following the gaps identification phase in the eTMF system in the organization case should develop proper practices, tools, and guidelines for success in system implementation using a knowledge management approach. Furthermore, an efficient knowledge management program allows the organization to manage and appropriately utilize different types of knowledge, improve productivity, and achieve satisfaction among employees by promoting continuous learning and development within the organization.

\section{REFERENCES}

[1] Association for Information and Image Management, "Glossary: What is Enterprise Content Management (ECM)?" 2017 [Online]. Available: goo.gl/6rcUUW

[2] R. O'Callaghan and M. Smits, "A strategy development process for enterprise content management," in Proceedings European Conference on Information Systems, Regensburg, Germany, 2005.

[3] J. A. Alalwan and H. R. Weistroffer, "Enterprise content management research: A comprehensive review," Journal of Enterprise Information Management, vol. 25, no. 5, pp. 441-461, 2012.

DOI: $10.1108 / 17410391211265133$

[4] J. Sprehe, "The positive benefits of electronic records management in the context of enterprise content management," Government Information Quarterly, vol. 22, no. 2, pp. 297-303, 2005.

DOI: $10.1016 /$ j.giq.2005.02.003

[5] B. T. Blair, "An enterprise content management primer, Information Management Journal, vol. 38, no. 5, pp. 64-66, 2004 . DOI: 10.1016/b978-0-08-100874-4.00005-3

[6] E. Ammenwerth, S. Gräber, G. Herrmann, T. Bürkle and J. König, "Evaluation of health information systems-problems and challenges," International Journal of Medical Informatics, vol. 7, no. 3-2, pp. 125-135, 2003. DOI: $10.1016 / \mathrm{s} 1386-5056(03) 00131-\mathrm{x}$

[7] H. S. Ahmad, I. M. Bazlamit and M. D. Ayoush, "Investigation of document management systems in small size construction companies in Jordan," Procedia Engineering, vol. 182, pp. 3-9, 2017.

DOI: $10.1016 /$ j.proeng.2017.03.101

[8] C. S. Venter, "An overview of the importance of the Trial Master File (TMF) and the required contents in clinical trials as stipulated in the ICH GCP guideline," Texila International Journal of Clinical Research, vol. 3, no. 2, pp. 1-4, 2016. DOI: 10.21522/tijcr.2014.03.02.art009

[9] R. Sama and N. V. Lakshmi, "Electronic Trial Master File (eTMF): An indispensable tool that collects and files es- 
sential documents of a clinical trial: A review," International Journal of Research and Development in Pharmacy and Life Sciences, vol. 6, no. 1, pp. 2476-2473, 2016.

[10] A. Grund and J. Mielebacher, "Electronic trial master file: Gaining efficiency for oversight and control," Journal for Clinical Studies, vol. 7, no. 4, pp. 22-25, 2006.

[11] A. Hecht, B. Busch-Heidger, H. Gertzen, H. Pfister, B. Ruhfus, P. H. Sanden and G. B. Schmidt, "Quality expectations and tolerance limits of Trial Master Files (TMF): Developing a risk-based approach for quality assessments of TMFs," German Medical Science, vol. 13, pp. $1-5,2015$
[12] R. S. Barbour, "Checklists for improving rigour in qualitative research: A case of the tail wagging the dog?" BMJ Clinical Research, vol. 322, pp. 1115-1117, 2001.

[13] H. E. McNay, "Enterprise content management: An overview," in Proceedings Professional Communication Conference, Portland, Oregon, 2002.

[14] S. Nordheim and T. Päivärinta, "Customization of enterprise content management systems: An exploratory case study," in Proceedings The 37th Hawaii International Conference on System Sciences, Big Island, HI, 2004. 